\title{
On the Recovery of Terrestrial Wireless Network using Cognitive UAVs in the Disaster Area
}

\author{
Najam Ul Hasan ${ }^{1}$, Prajoona Valsalan ${ }^{2}$, Umer Farooq ${ }^{3}$, Imran Baig*4 \\ Department of Electrical and Computer Engineering \\ Dhofar University, Salalah \\ Sultanate of Oman
}

\begin{abstract}
Natural disasters such as earthquakes, floods and fires may cause the existing wireless network infrastructure to collapse, leaving behind several disconnected network parts. UAVs could help to establish communication between these disconnected parts using their ability to hover and fly across the affected region. However, UAV deployment faces several problems, including how many UAVs would be sufficient and where they could be placed. Such problems can be addressed centrally in a situation with verified information about the segmented network, such as the number of disconnected parts, the number of nodes in each part and the location of each node. However, a damaged network with unknown information (which is mostly the case) requires a distributed networking establishment mechanism. Therefore, this paper proposes an algorithm to restore connectivity among the disconnected parts of the damaged network. Cognitive radiobased UAVs (CR-UAVs) fly into the affected area and try to connect the various parts of the damaged network using the proposed algorithm. The main objective of the proposed algorithm is to connect the different disconnected parts of the broken network with the fewest possible UAVs in the least possible time. The results of the MATLAB simulation illustrate the significance of the proposed algorithm in terms of the number of UAVs used and the total distance they fly.
\end{abstract}

Keywords-Cognitive radio networks; spectrum allocation; sensor network

\section{INTRODUCTION}

Different natural disasters, including earthquakes, windstorms, hurricanes, floods and fires, can lead to a massive, unexpected loss of life and infrastructure. Although this kind of damage can not be completely avoided, but, it can be minimized. A key factor in this direction is the disaster management team's response time. However, this response time depends on how fast the disaster team can assess the situation in the concerned region. Therefore, data of the concerned region must be obtained as soon as possible. However, getting a ground view of the affected area is time-consuming and may not be feasible in most situations. So an aerial view might be a better and more effective alternative for these types of situations. One of the best and quickest way to obtain an aerial view is by using unmanned aerial vehicles (UAVs). The data obtained from UAVs may be useful in a number of disaster-related applications, including surveillance, forecasting, information sharing, rescue operations, evacuation assistance, and many more [1]. However, for all such applications, a communication network is required to disseminate the data obtained via UAVs to the concerned entities. UAV network communication is mainly divided into the following: (1) UAV to UAV; (2) UAV to remote station; and (3) UAV to terrestrial network in the region [2]. Each of these forms of communication has its own significance. UAV to UAV communication is necessary to perform assigned tasks in a more coordinated manner. UAVs to remote station communication can be helpful for the disaster management team to make their decisions. UAVs to terrestrial network communication in the affected area may be useful for federating the various disconnected parts of the damaged network. This paper focuses mainly on the communication of UAVs to the terrestrial network in the affected disaster area.

UAVs need to be deployed in the affected region to connect to terrestrial network devices. But there are some issues that need to be addressed here: First of all, how can UAVs find nodes that are still alive in the damaged network? Second, each alive node belongs to which part of the damaged network ? Third, how many UAVs are needed and where each of these UAV can be located to integrate the disconnected parts? In order to answer these questions, first, the UAVs are equipped with cognitive radio technology (CRT) to detect alive nodes in the damaged network. With the help of CRT, UAVs can search the spectrum to locate any node that is sending its signals at any frequency band [3]. And the UAVs equipped with the CRT are termed as the CR-UAV. Then, an antenna array can be mounted on UAVs that can help to determine the angle of arrival (AoA) for the received signal, eventually deciding that this node belongs to which part of the disconnected damaged network. After that, the UAVs present in the affected part determine how many nodes there are in each disconnected part, and the UAV closest to the disconnected part with the largest number of nodes move to provide connectivity to that part. Where the UAVs coordinates to determine the closest UAV on the basis of the received signal strength indicator (RSSI) from the received signal of the nodes of that disconnected part.The same procedure is repeated for the rest of the disconnected parts and for the remaining UAVs. And once all the UAVs present in the field have been engaged, the remote station can provide an additional UAV node, if necessary.

We proposed an algorithm to provide connectivity to the maximum possible live nodes in a damaged network using UAVs with their coordination and relocation. While relocating UAVs, the algorithm attempts to minimize both the number of UAVs and the distance flown by these UAVs so that desired connectivity can be achieved as soon as possible. The proposed algorithm enables UAVs to be deployed in groups to reduce the number of UAVs used rather than using all available UAVs at once. The next group is sent to the field, once the previous group has been completely deployed and additional UAVs are needed. However, the number of UAVs in the next group may 
vary based on information obtained from the UAVs already deployed in the disaster area. All UAVs share their current position and move in a way that ensures that the disconnected parts are connected within a shorter time period. A brief summary of this work is provided as follows.

- A strategy is introduced for the identification of alive nodes in the destroyed wireless network in a disaster area and a problem for restoring communication between these nodes with the help of UAV is formulated.

- An algorithm is proposed to determine the location of each UAV to connect all disconnected parts of the damaged network while reducing the UAV used and the total distance flown for all UAVs to do so.

- Matlab simulations are performed to demonstrate the significance of the proposed algorithm in terms of the number of UAVs used and the total distance flown by all UAVs.

The rest of the paper is organized as follows. The related work is presented in Section II. The system model and the problem are defined in Section III. The proposed solution is presented in Section IV. Section V shows the results of Matlab simulations and Section VI concludes the paper.

\section{RELATED WORK}

Over the past few years, various research has been done on the use of UAVs for disaster management. The previous work on connectivity of UAVs can be categorized into two types that is air to air communication and air to ground communication. Both of these communication are equally important for the success of the UAVs applications. In [4], the authors present the latest developments in the use of UAVs for network-assisted first disaster management response, and identify issues remaining to be resolved. There are many other reviews and research articles on this topic; however, very little work has been done on the use of CR-UAVs for managing disasters. The first survey paper on integrating CRT with UAVs is [5]. In addition to presenting different issues and challenges for the CRT-based UAVs, the authors discuss the use of these UAVs for various applications, including disaster management. In subsequent papers, the use of CR-UAVs in disaster situations is given more attention. For example, [6] investigates ways of improving the communication of CRTbased UAVs over the unlicensed spectrum using a proposed spectrum-sensing architecture. Likewise, in [7], the authors present a prototype of a CRT-based UAV aerial spectrummonitoring system. This system scans a wide range of spectra and measures the signal strength and other related parameters for the captured signals. In [8], the authors discuss a new scenario of spectrum sharing between CRT-based UAVs and wireless terrestrial communication systems. A mechanism is introduced to improve UAV communication by means of power, mobility, and trajectory control. In [9], the authors present an adaptive error control framework to disseminate data on a CRT-based UAV network in order to improve energy efficiency. The authors of [10] propose a method for clustering different UAVs in a CRT-based UAV network based on an available common channel. All of the above work focuses on improving UAV-to-UAV communication and UAV-to-remotestation communication. What makes our work different is that

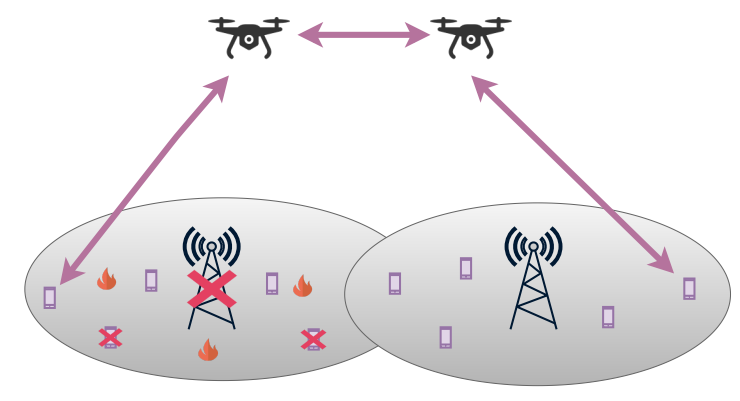

Fig. 1. Federation of a Damaged Terrestrial Wireless Network using UAVs.

we focus on UAV-to-terrestrial-wireless-network communication.

\section{System Model And Problem Statement}

\section{A. System Model}

Consider a geographical area in which $\mathrm{N}$ node terrestrial wireless network has been operating since a certain timespan. Suppose there is a disaster in this region that destroys the $\mathrm{F}$ nodes of this network. However, some nodes may still work correctly, leading to a different $\mathrm{K}$ disconnected part of this network. Although all alive nodes of a disconnected part may be connected, but, these nodes are unable to communicate with the alive nodes of any other disconnected part. Consequently, the entire network may no longer be usable unless the connection between the disconnected parts is restored. To restore the required connections as quickly as possible, M UAVs are sent to this area, which can integrate the disconnected parts into one unit. To achieve this goal, a number of assumptions have been made; first, UAVs with the help of CRT locate nodes that are still working properly; second, UAVs are thought to be rich in memory, computing and power resources; third, all UAVs are interconnected and can coordinate; and fourth, no prior information is provided about the damaged network. Due to this situation, UAVs must fly across this region to find a node that is working properly and act as a relay node to pass its data to other nodes. When federating disconnected parts, we have two goals: first, to use fewer UAVs; and second, to reduce the distance flown by UAVs so that the recovery process can be completed in less time. Fig. 1 shows the possible configuration of the disaster situation with the damaged infrastructure.

\section{B. Problem Statement}

There are $\mathrm{N}$ nodes in the network so that any node can communicate directly or indirectly with another node within the network. Let us assume that $\mathrm{F}$ nodes are destroyed leaving behind N-F functional nodes. This may lead to two or more disconnected parts of the existing wireless network. To restore the communication between these disconnected parts, M UAVs are sent to this region. These UAVs communicate with each other to position them in such a way that the disconnected parts are federated into a single network. There are two main objectives while doing so

1) Minimize the number of UAVs used to establish the connectivity. 
2) Minimize the total distance D flown by UAVs used to restore the connectivity. The distance $\mathrm{D}$ can be mathematically written as follows

$$
D=\sum_{i=1}^{M} d_{i}
$$

Where $d_{i}$ is the distance flown by the ith UAV.

\section{PROposed SOLUTION}

Our proposed solution consists of three steps to solve the problem described in the previous section. First, UAVs are sent to the affected area. Second, UAVs can identify the properly working nodes by listening to various frequencies. Third, the UAVs coordinate to move them to connect different parts of the network that are disconnected.

Once UAVs have flown into the disaster area, using AoA and RSSI, each UAV estimates the approximate number of nodes in the jth disconnected part denoted by $x_{i j}$. As the number of nodes estimated by each UAV depends on RSSI and AoA, the value of estimated numbers of nodes by any two UAVs in the jth disconnected part may or may not be the same. Therefore, each UAV calculates the accumulative number of the approximate node denoted by $T_{j}$ in each jth disconnected part using received $x_{i j}$ from the other (M-1) UAVs which can be expressed as follows:

$$
T_{j}=\sum_{i=1}^{M} x_{i j}
$$

Based on $T_{j}$, a probability density function for each $\mathrm{jth}$ disconnected part is computed as:

$$
P_{j}=\frac{T_{j}}{\sum_{j=1}^{K} T_{j}}
$$

where $\mathrm{K}$ is the total number of disconnected parts. K's value is pre-assumed based on the disaster scale.

After determining the value of $P_{j}$ for each jth disconnected part, each UAV sorts $P_{j}$ in descending order to finds out the part with the highest value of $P_{j}$. Then, UAVs cooperate to find the node $\mathrm{u}$ of the highest $P_{j}$ segment, with a minimum distance to any of the mth UAV that is denoted by $d_{m u}$. The distance $d_{m u}$ is calculated on the basis of the RSSI information obtained by $\mathrm{m}$ from $\mathrm{u}$ using the following formula given in [11] as follows

$$
d_{m u}=10^{\frac{R S S I-C}{-10 \times \alpha}}
$$

Where $\alpha$ is the exponent of the path loss and its value for the outdoor environment is 2.1 . C is considered to be a fixed constant and has a value of 6.9. If $d_{m u}>d$, the mth UAV is relocated so that its distance from the node $u$ is less than $d$. Where $\mathrm{d}$ is the fixed distance between any two nodes to retain the connectivity between them. Once the mth UAV is relocated, it is assumed to be part of the jth disconnected part. So it is removed from the list of UAVs. When it comes to connecting this UAV to other UAVs, there is no issue as it is assumed that each UAV can communicate directly or indirectly irrespective of how far they are from each other. The same process is repeated for each disconnected part according to the sorted order. UAVs are deployed in groups; the next UAV group will be deployed if the UAVs deployed in the previous group are not sufficient. The pseudo code of the proposed algorithm is given as follows:

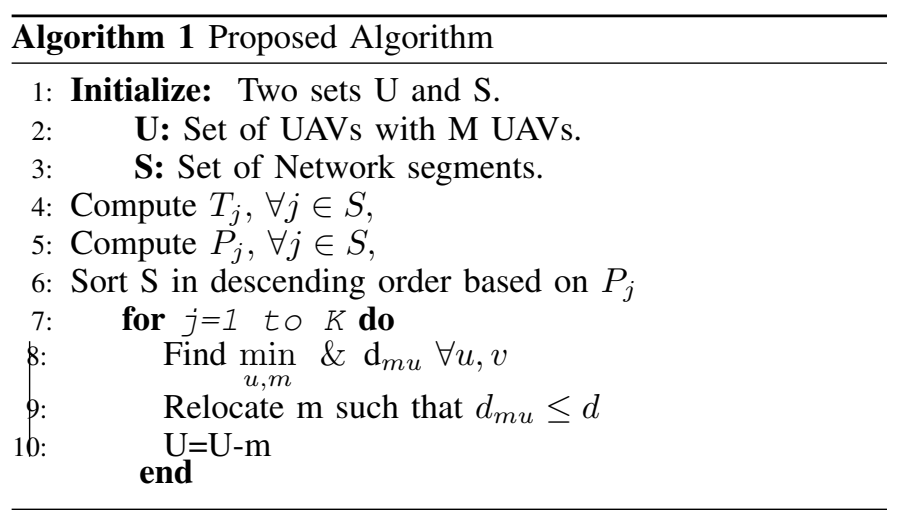

\section{Simulation Results}

\section{A. Simulation Setup}

In order to evaluate the performance of the proposed algorithm, we consider a damaged wireless network with $\mathrm{N}$ alive nodes in a geographical area. Initially, a CR-based UAVs group is deployed close to the center of the region. We assumed that the initially deployed group had a number of CR-based UAVs fixed to five for our simulation. However, it may vary depending on the area of the affected region. After that, if a further UAV is needed, only one additional UAV fly to the affected region. However, this can also vary depending on the real-time information that already deployed UAVs obtain from the affected area. The damaged wireless network is divided into a fixed number of disconnected $\mathrm{K}$ networks based on AoA information collected using the UAVmounted antenna array. If the distance between a UAV and a node is less than $70 \mathrm{~m}$, they are assumed to be connected. Based on the aforementioned parameters setting, a number of cases are considered for the simulation with variation in the size of the geographical area, the number of alive nodes (N-F) and the number of disconnected network (K). For each case hundreds of different topologies are evaluated and the average is reported. For the performance evaluation, two parameters are considered, i.e. 1) the total distance traveled by UAVs denoted by $\mathrm{D}$ and 2) the total number of UAVs (M) deployed.

Fig. 2 shows the total distance D flown by UAVs versus the density of alive (N-F) nodes in order to federate different disconnected parts of the damaged network. In the damaged network, the number of alive node varies from 20 to 40 . In the first group, the number of UAVs deployed is 5 . The average result is shown for 100 different topologies. In addition, the simulation is performed for areas with sizes $600 \times 600$ and $800 \times 800 m_{2}$ respectively. First, as the number of alive nodes increases, the distance D decreases, implying that the UAV needs to fly less to connect the different disconnected parts. However, with the increase in the size of the considered area, UAVs had to fly more to restore communications among the disconnected parts.

Fig. 3 shows how many UAVs are required to restore connectivity versus the number of alive nodes. As the value of 


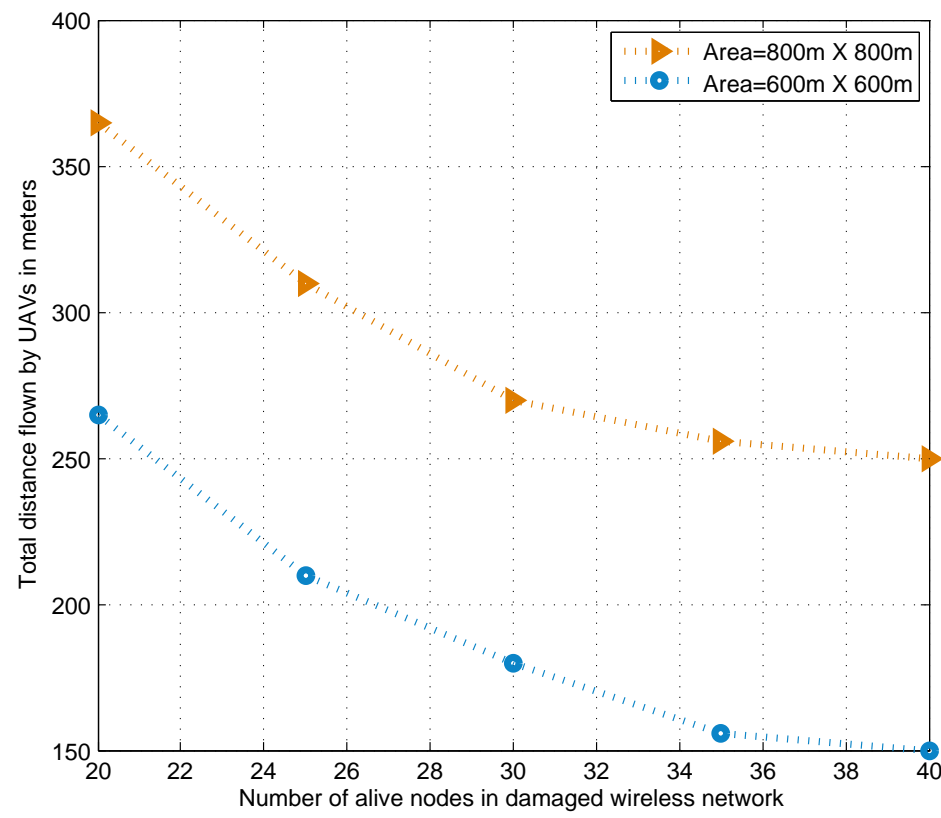

Fig. 2. Total Distance Flown by UAVs vs Alive Nodes.

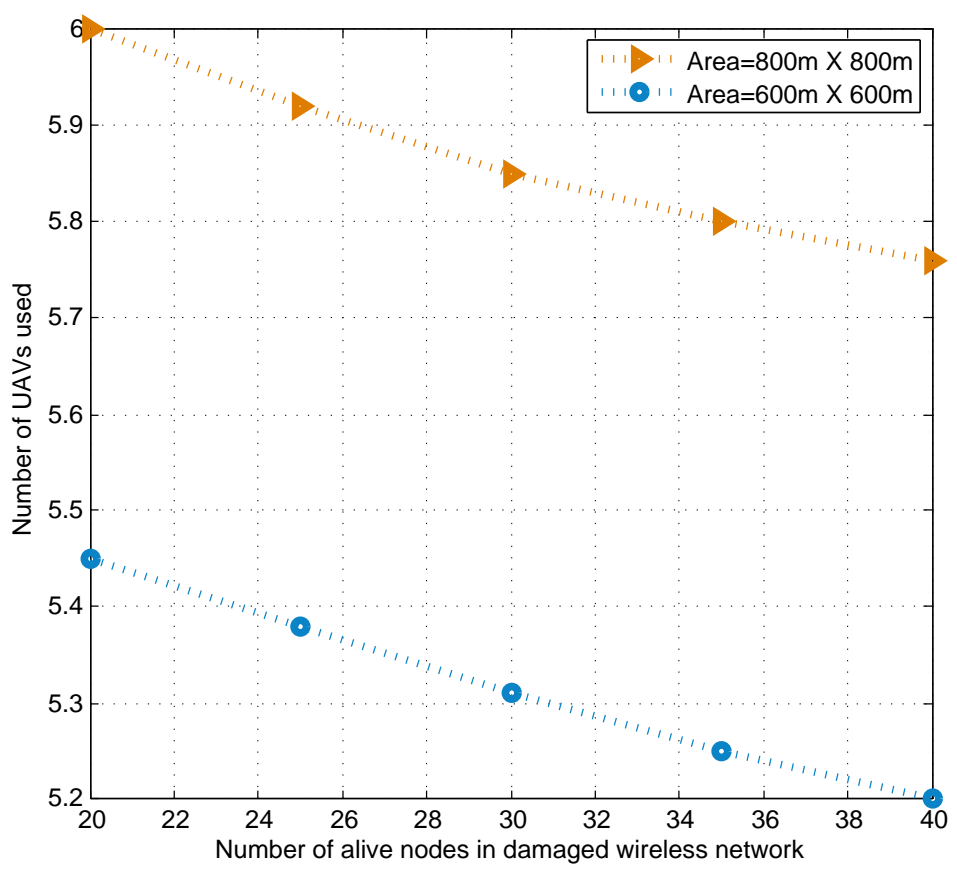

Fig. 3. Total used UAVs vs Alive Nodes.

alive nodes increases in the damaged network, the number of used UAVs decreases, this means that fewer UAVs are needed to restore the connection between the disconnected parts of the damaged network. However, more UAVs are needed with a wider geographic area.

\section{CONCLUSION}

In this paper, we proposed an algorithm for the recovery of the damaged terrestrial wireless network in a disaster zone. The algorithm aims at lowering recovery costs, not only by reducing the number of UAVs used, but also by reducing their movements for the connectivity. The approach uses the AoA data collected by an antenna array mounted at the top of the UAVs to divide the damaged node into different disconnected parts and the RSSI value to determine the relative distance between a UAV and any alive node. The solution moves UAVs to point where the distance is less than 70 meters between the UAV and any node of a particular disconnected part that it wants to connect. Hundreds of different topologies are being evaluated to determine the performance of the proposed algorithm and the findings are shown in terms of the UAVs used and their total distance flown. Our work has a limitation that only one UAV is moved at a time, but in the future this work can be further extended to the simultaneous movement of multiple UAVs to recover the connectivity with in much lesser time.

\section{REFERENCES}

[1] H. Hildmann and E. Kovacs, Using Unmanned Aerial Vehicles (UAVs) as Mobile Sensing Platforms (MSPs) for Disaster Response, Civil Security and Public Safety, Drones, 3(59), 2019.

[2] B. Li, Z. Fei, and Y. Zhang, UAV Communications for 5G and Beyond: Recent Advances and Future Trends, IEEE Internet of Things Journal, 6(2), 2241 - 2263, 2019.

[3] G. M. D. Santana ; R. S. Cristo , Catherine Dezan, Jean-Philippe Diguet, etal.Cognitive Radio for UAV communications: Opportunities and future challenges, in proc. of IEEE International Conference on Unmanned Aircraft Systems (ICUAS), june 12-15, 2018.

[4] M. Erdelj, E. Natalizio, K. R. Chowdhury, and I. F. Akyildiz, Help from the Sky: Leveraging UAVs for Disaster Management, IEEE Pervasive Computing, 16(1), 2017.

[5] Y. Saleem, M.H. Rehmani, and S. Zeadally, Integration of Cognitive Radio Technology with Unmanned Aerial Vehicles: Issues, Opportunities, and Future Research Challenges, Journal of Network and Computer Applications, Volume 50, Pages 15-31, April 2015.

[6] Jacob, P.; Sirigina, R.P.; Madhukumar, A.; Prasad, V.A. Cognitive radio for aeronautical communications: A survey. IEEE Access 2016, 4, 3417-3443.

[7] W. T. Chen, C. H. Ho, Spectrum monitoring with unmanned aerial vehicle carrying a receiver based on the core technology of cognitive radio - A software-defined radio design, Journal of Unmanned Vehicle Systems, 2017, Vol. 5, No. 1 : pp. 1-12.

[8] Y. Huang, J. Xu, and R. Zhang, Cognitive UAV Communication via Joint Trajectory and Power Control, in proc. of IEEE 19th International Workshop on Signal Processing Advances in Wireless Communications (SPAWC), June 25-28, 2018.

[9] N. U. Hasan, W. Ejaz, U. Farooq, I. Baig and M. Zghaibeh, Adaptive Error Control Framework for a Multihop Cognitive Radio based UAVs for Disaster Management, in proc. of IEEE 5th International Conference on Mechatronics System and Robots (ICMSR), 3-5 May, 2019.

[10] N. U. Hasan, M. Zghaibeh, W. Ejaz, A. Shahid, and A. Anpalagan, On Provision of Resilient Connectivity in Cognitive Unmanned Aerial Vehicles, in proc. of IEEE International Conference on Communications Workshops (ICC Workshops), May 23-25, 2019.

[11] O. G. Adewumi, K. Djouani, and A. M. Kurien, "RSSI based indoor and outdoor distance estimation for localization in WSN," in Industrial Technology (ICIT), 2013 IEEE International Conference on, 2013, pp. 1534-1539. 